\title{
Identification of structure-controlling rice biosynthesis enzymes
}

Jihui Zhu, ${ }^{\dagger \dagger}$ Changquan Zhang, ${ }^{\dagger, \S}$ Jianlong Xu, Robert G. Gilbert**\$, Qiaoquan $\mathrm{Liu}^{\dagger \dagger, \S}$

${ }^{\dagger}$ Jiangsu Key Laboratory of Crop Genomics and Molecular Breeding, Key Laboratory of Plant Functional Genomics of the Ministry of Education, College of Agriculture, Yangzhou University, Yangzhou, Jiangsu, 225009, China

${ }^{\ddagger}$ The University of Queensland, Centre for Nutrition and Food Sciences, Queensland Alliance for Agriculture and Food Innovation, Brisbane, QLD, 4072, Australia

${ }^{\S}$ Jiangsu Key Laboratory of Crop Genetics and Physiology, Joint International Research Laboratory of Agriculture and Agri-Product Safety, Co-Innovation Center for Modern Production Technology of Grain Crops of Jiangsu, Yangzhou University, Yangzhou, Jiangsu, 225009, China

"Institute of Crop Sciences, Chinese Academy of Agricultural Sciences, Beijing, 9 100081, China

* Corresponding authors: Prof. RG Gilbert, b.gilbert@uq.edu.au; Prof. Q Liu, qqliu@yzu.edu.cn 
Flow diagram to find correlations between isoforms and starch biosynthesis parameters.

An example from Fig. 5a, isoform SSI:

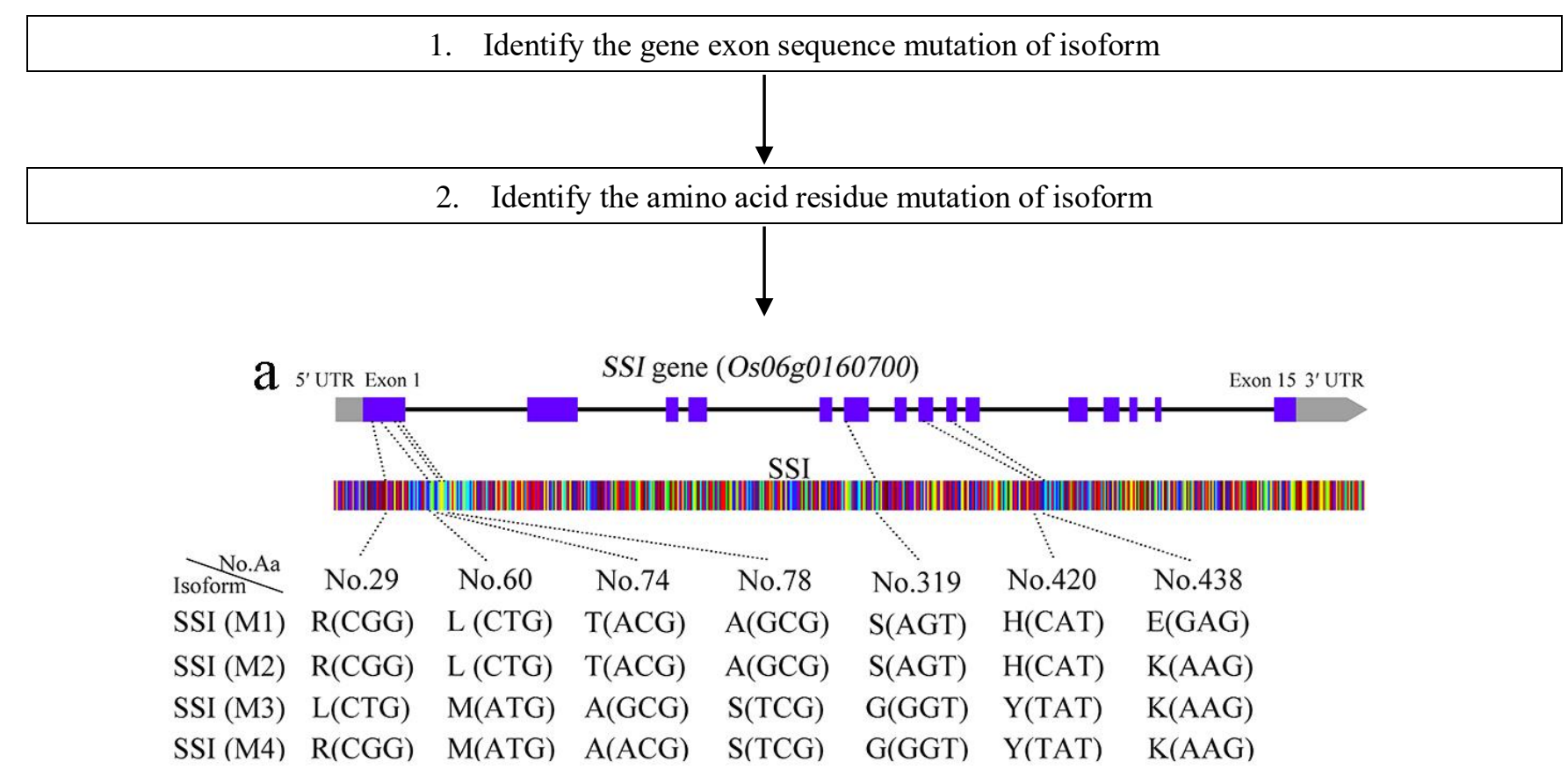

Fig. 5a. The gene and enzyme mutant group differences of SSI. SSIM1, "SSI" represents SSI isoform, "M1" represents the isoform type 1. The M1, M2, M3 and M4 indicate four different isoform types.

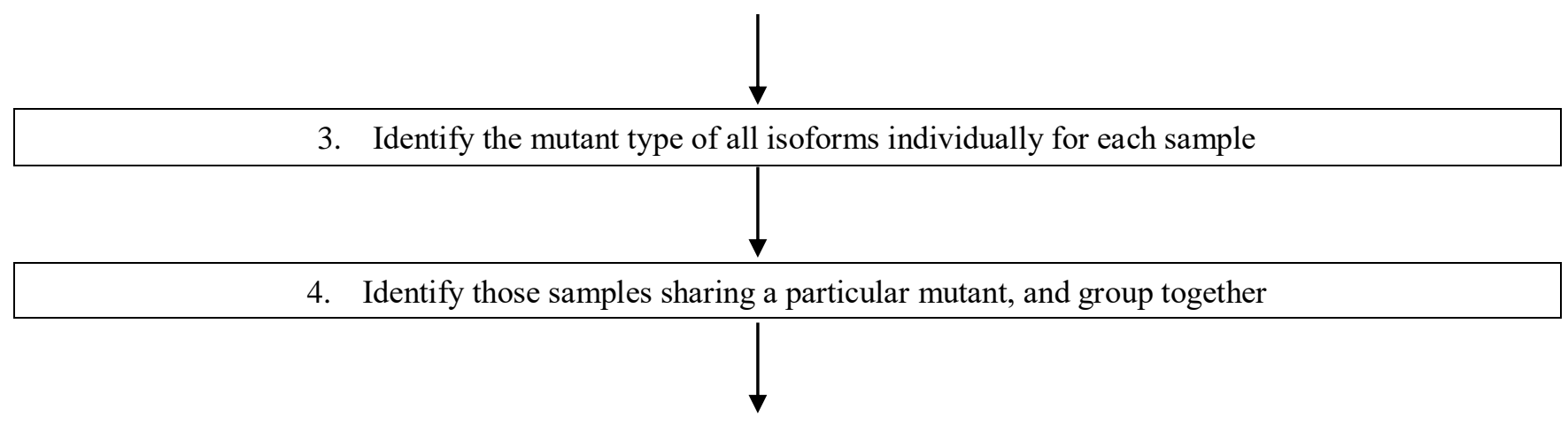


Table 1 Four SSI isoform types detected from all 87 samples with fitted starch biosynthesis parameters.

\begin{tabular}{|c|c|c|c|c|c|c|c|c|c|c|c|}
\hline $\begin{array}{c}\text { Sample } \\
\text { name }\end{array}$ & $\begin{array}{c}\text { Isoform } \\
\text { type }\end{array}$ & $\begin{array}{r}h_{\mathrm{Ap}, \mathbf{i}} \\
\times 10^{3}\end{array}$ & $\begin{array}{r}h_{\mathrm{Ap}, \mathrm{iii}} \\
\times 10^{3}\end{array}$ & $\begin{array}{l}h_{\mathrm{Ap}, \mathrm{v}} \\
\times 10^{3}\end{array}$ & $\begin{array}{l}h_{\mathrm{Am}, \mathrm{i}} \\
\times 10^{3}\end{array}$ & $\begin{array}{r}h_{\mathrm{Am}, \mathrm{ii}} \\
\times 10^{3}\end{array}$ & $\begin{array}{l}\beta_{\mathrm{Ap}, \mathrm{i}} \\
\times 10^{3}\end{array}$ & $\begin{array}{r}\beta_{\text {Ap,iii }} \\
\times 10^{3}\end{array}$ & $\begin{array}{l}\beta_{\mathrm{Ap}, \mathrm{v}} \\
\times 10^{3}\end{array}$ & $\boldsymbol{\beta}_{\mathrm{Am}, \mathrm{i}}$ & $\boldsymbol{\beta}_{\mathrm{Am}, \mathrm{ii}}$ \\
\hline CH1063 & SSIM1 & 1031.00 & 25.96 & 1.12 & 59.93 & 180.47 & 107.83 & 43.36 & 9.84 & 136.69 & 22.04 \\
\hline G562 & SSIM1 & 1095.70 & 26.23 & 1.45 & 143.55 & 123.14 & 100.48 & 42.41 & 11.64 & 93.84 & 32.53 \\
\hline C351 & SSIM1 & 1098.60 & 26.63 & 1.00 & 47.49 & 170.32 & 101.06 & 44.02 & 11.63 & 113.42 & 23.01 \\
\hline C38 & SSIM1 & 1000.00 & 25.00 & 0.16 & 0.00 & 0.00 & 99.00 & 46.60 & $\mathrm{~N}$ & 0.00 & 0.00 \\
\hline G426 & SSIM1 & 1014.30 & 24.22 & 0.64 & 31.98 & 104.46 & 101.79 & 45.34 & 10.75 & 136.73 & 29.86 \\
\hline C969 & SSIM1 & 1011.80 & 25.81 & 1.01 & 57.45 & 144.76 & 99.46 & 43.20 & 10.39 & 122.49 & 34.90 \\
\hline SE389 & SSIM1 & 963.59 & 26.16 & 1.44 & 97.22 & 74.39 & 99.79 & 43.45 & 14.97 & 110.29 & 25.33 \\
\hline C1061 & SSIM1 & 1030.00 & 26.81 & 1.19 & 57.90 & 180.37 & 98.97 & 43.09 & 12.84 & 115.92 & 22.47 \\
\hline G584 & SSIM1 & 894.11 & 24.99 & 2.13 & 0.00 & 0.00 & 101.82 & 40.38 & 18.90 & 0.00 & 0.00 \\
\hline SE217 & SSIM1 & 1060.30 & 25.78 & 1.03 & 52.65 & 190.84 & 98.22 & 42.56 & 9.99 & 110.20 & 24.09 \\
\hline G357 & SSIM1 & 1040.00 & 27.20 & 2.46 & 237.40 & 38.94 & 101.00 & 40.60 & 15.00 & 141.43 & 55.67 \\
\hline C278 & SSIM1 & 1080.00 & 25.70 & 0.53 & 60.60 & 164.50 & 96.50 & 40.10 & 28.10 & 117.14 & 24.16 \\
\hline C291 & SSIM1 & 994.89 & 27.15 & 2.40 & 212.95 & 30.36 & 101.63 & 41.37 & 15.83 & 139.66 & 45.34 \\
\hline SE108 & SSIM2 & 1080.80 & 25.03 & 0.84 & 41.24 & 131.22 & 99.95 & 43.02 & 10.18 & 133.31 & 21.38 \\
\hline G220 & SSIM2 & 1022.20 & 27.98 & 0.60 & 22.33 & 40.98 & 105.62 & 46.73 & 13.41 & 126.38 & 19.59 \\
\hline C1196 & SSIM2 & 1037.80 & 23.99 & 0.54 & 34.22 & 88.11 & 102.31 & 45.72 & 10.19 & 135.37 & 19.81 \\
\hline C1170 & SSIM2 & 1024.20 & 26.11 & 2.21 & 214.13 & 90.26 & 100.84 & 41.95 & 12.52 & 214.62 & 103.19 \\
\hline G436 & SSIM2 & 1010.00 & 27.40 & 0.95 & 30.78 & 127.79 & 99.60 & 44.80 & 16.40 & 162.71 & 19.53 \\
\hline C1018 & SSIM2 & 1027.90 & 24.81 & 0.56 & 18.61 & 66.80 & 100.20 & 45.63 & 9.84 & 124.83 & 22.44 \\
\hline CH1091 & SSIM2 & 942.96 & 25.25 & 0.52 & 25.66 & 97.76 & 105.46 & 45.19 & 10.17 & 139.65 & 20.04 \\
\hline C53 & SSIM2 & 1094.20 & 23.78 & 0.89 & 44.73 & 143.84 & 101.12 & 42.92 & 10.31 & 114.32 & 24.20 \\
\hline CH1117 & SSIM2 & 1027.70 & 25.27 & 2.32 & 207.06 & 47.42 & 103.04 & 41.86 & 12.90 & 139.81 & 60.82 \\
\hline G97 & SSIM2 & 1010.00 & 27.40 & 1.34 & 54.88 & 225.36 & 106.00 & 44.70 & 16.60 & 138.80 & 30.16 \\
\hline G528 & SSIM2 & 1084.60 & 24.95 & 0.83 & 40.29 & 152.64 & 102.07 & 44.57 & 10.27 & 130.42 & 21.72 \\
\hline G175 & SSIM2 & 1058.70 & 25.79 & 1.64 & 158.52 & 106.22 & 100.56 & 42.31 & 9.84 & 97.61 & 40.13 \\
\hline G350 & SSIM2 & 1099.10 & 24.91 & 0.62 & 46.67 & 154.40 & 101.66 & 43.71 & 9.99 & 148.79 & 21.87 \\
\hline G242 & SSIM2 & 919.86 & 23.81 & 0.51 & 28.86 & 80.24 & 108.46 & 45.92 & 17.12 & 163.03 & 20.70 \\
\hline G186 & SSIM2 & 935.06 & 24.78 & 0.53 & 24.26 & 98.63 & 108.63 & 46.29 & 18.61 & 133.60 & 20.36 \\
\hline G239 & SSIM2 & 1013.70 & 24.42 & 0.53 & 23.23 & 83.10 & 102.25 & 46.41 & 16.00 & 113.13 & 21.17 \\
\hline C1038 & SSIM2 & 865.34 & 24.72 & 1.16 & 60.10 & 9.13 & 99.49 & 44.23 & 20.44 & 162.49 & 45.73 \\
\hline G194 & SSIM2 & 1003.50 & 26.35 & 1.23 & 143.25 & 50.90 & 100.76 & 44.45 & 21.61 & 112.79 & 28.44 \\
\hline G577 & SSIM2 & 1033.50 & 24.57 & 0.31 & 21.03 & 102.35 & 99.98 & 44.01 & 33.86 & 122.57 & 21.17 \\
\hline SE91 & SSIM2 & 1027.30 & 26.29 & 0.87 & 40.10 & 109.78 & 100.40 & 47.73 & 18.13 & 128.60 & 23.83 \\
\hline G649 & SSIM2 & 947.78 & 28.59 & 2.76 & 260.87 & 75.99 & 101.45 & 41.86 & 15.36 & 132.95 & 57.54 \\
\hline SE14 & SSIM2 & 1011.60 & 24.70 & 0.66 & 33.35 & 109.13 & 101.82 & 45.33 & 17.65 & 131.68 & 28.36 \\
\hline G224 & SSIM2 & 999.16 & 24.98 & 1.02 & 80.95 & 78.04 & 100.59 & 45.05 & 11.06 & 94.40 & 34.07 \\
\hline G520 & SSIM2 & 881.75 & 24.59 & 0.60 & 10.37 & 14.87 & 98.86 & 46.54 & 26.49 & 208.07 & 67.25 \\
\hline C1031 & SSIM2 & 1044.50 & 23.71 & 0.51 & 28.57 & 110.00 & 102.92 & 45.60 & 10.44 & 106.21 & 16.81 \\
\hline G222 & SSIM2 & 1016.40 & 24.61 & 0.61 & 44.21 & 147.81 & 102.41 & 45.90 & 9.99 & 155.34 & 20.13 \\
\hline G157 & SSIM2 & 979.68 & 29.20 & 0.77 & 33.98 & 110.75 & 96.80 & 45.38 & 15.89 & 135.39 & 20.45 \\
\hline G245 & SSIM2 & 891.00 & 28.80 & 0.64 & 43.02 & 163.57 & 104.00 & 42.70 & 15.50 & 138.21 & 25.58 \\
\hline CH1002 & SSIM2 & 1011.90 & 24.44 & 0.74 & 40.27 & 113.94 & 101.76 & 45.31 & 12.46 & 169.29 & 21.31 \\
\hline C1128 & SSIM2 & 1013.40 & 24.67 & 0.68 & 32.08 & 117.18 & 102.44 & 46.46 & 13.26 & 138.03 & 22.17 \\
\hline SE342 & SSIM2 & 1020.70 & 25.08 & 0.65 & 37.06 & 93.44 & 102.84 & 46.24 & 13.10 & 161.05 & 21.30 \\
\hline
\end{tabular}




\begin{tabular}{|c|c|c|c|c|c|c|c|c|c|c|c|}
\hline G561 & SSIM2 & 1034.40 & 24.00 & 0.57 & 41.43 & 98.05 & 100.26 & 43.66 & 16.53 & 123.48 & 17.54 \\
\hline CH1148 & SSIM2 & 1020.00 & 26.60 & 1.10 & 60.54 & 199.96 & 103.00 & 44.40 & 18.20 & 125.25 & 21.89 \\
\hline G246 & SSIM2 & 937.00 & 26.70 & 0.70 & 57.53 & 155.50 & 102.00 & 47.90 & 14.60 & 156.45 & 24.42 \\
\hline SE461 & SSIM2 & 1026.70 & 23.39 & $\mathrm{~N}$ & 0.00 & 0.00 & 100.79 & 45.44 & $\mathrm{~N}$ & 0.00 & 0.00 \\
\hline $\mathrm{C} 42$ & SSIM2 & 978.30 & 24.76 & 0.83 & 44.18 & 156.33 & 101.54 & 44.80 & 13.95 & 130.70 & 19.49 \\
\hline SE223 & SSIM2 & 1055.70 & 25.11 & 0.73 & 37.79 & 115.67 & 100.15 & 43.23 & 10.01 & 131.78 & 21.08 \\
\hline SE228 & SSIM2 & 980.07 & 25.53 & 1.86 & 175.88 & 8.40 & 103.33 & 41.70 & 27.55 & 195.12 & 42.50 \\
\hline SE372 & SSIM2 & 1100.00 & 25.20 & 0.95 & 57.18 & 144.80 & 99.46 & 42.06 & 10.56 & 123.20 & 20.85 \\
\hline G101 & SSIM2 & 1086.40 & 25.04 & 0.01 & 31.85 & 137.42 & 98.92 & 41.59 & 29.99 & 124.51 & 23.81 \\
\hline C1221 & SSIM2 & 1108.00 & 24.63 & 0.41 & 36.71 & 125.95 & 99.85 & 42.07 & 22.96 & 140.47 & 21.14 \\
\hline SE344 & SSIM2 & 1043.20 & 25.08 & 0.47 & 37.47 & 90.83 & 100.31 & 44.21 & 10.01 & 168.23 & 19.67 \\
\hline G582 & SSIM2 & 982.44 & 24.33 & 0.91 & 97.11 & 70.59 & 100.95 & 44.68 & 15.89 & 95.49 & 34.31 \\
\hline G182 & SSIM3 & 1070.10 & 30.16 & 1.07 & 54.93 & 170.66 & 97.04 & 43.97 & 12.24 & 127.05 & 24.05 \\
\hline C1200 & SSIM3 & 1101.90 & 25.48 & 1.06 & 69.13 & 182.21 & 100.34 & 42.52 & 13.52 & 182.38 & 24.31 \\
\hline C125 & SSIM3 & 1092.00 & 25.53 & 1.31 & 47.31 & 172.96 & 100.01 & 42.04 & 10.77 & 112.20 & 24.12 \\
\hline C142 & SSIM3 & 1010.00 & 25.30 & 1.00 & 57.76 & 177.14 & 106.00 & 41.90 & 10.00 & 133.34 & 23.08 \\
\hline G546 & SSIM3 & 1003.60 & 26.44 & 1.73 & 168.95 & 110.18 & 107.24 & 42.24 & 10.82 & 110.80 & 38.99 \\
\hline C287 & SSIM3 & 1009.90 & 25.87 & 2.30 & 259.51 & 49.19 & 102.73 & 41.32 & 16.31 & 207.64 & 104.28 \\
\hline $\mathrm{C} 403$ & SSIM3 & 1097.20 & 24.37 & 1.08 & 89.25 & 208.47 & 99.38 & 40.43 & 10.03 & 114.88 & 29.39 \\
\hline C602 & SSIM3 & 1014.40 & 26.33 & 1.16 & 60.09 & 228.43 & 105.95 & 45.68 & 20.05 & 121.86 & 21.84 \\
\hline C266 & SSIM4 & 885.72 & 15.58 & 0.07 & 0.00 & 0.00 & 108.39 & 53.61 & 25.86 & 0.00 & 0.00 \\
\hline CH1099 & SSIM4 & 1097.00 & 25.80 & 1.13 & 38.75 & 163.66 & 100.42 & 42.44 & 10.03 & 113.33 & 21.14 \\
\hline CH1210 & SSIM4 & 1046.80 & 27.05 & 2.37 & 233.75 & 76.86 & 100.37 & 40.66 & 11.51 & 136.09 & 55.28 \\
\hline G360 & SSIM4 & 1104.10 & 25.33 & 1.03 & 45.86 & 200.02 & 101.40 & 42.40 & 10.36 & 125.29 & 29.73 \\
\hline $\mathrm{C} 272$ & SSIM4 & 1088.40 & 26.00 & 1.15 & 59.98 & 189.87 & 99.60 & 41.34 & 9.96 & 134.01 & 22.77 \\
\hline C1030 & SSIM4 & 1040.70 & 26.40 & 2.62 & 226.06 & 36.52 & 102.82 & 41.74 & 13.44 & 134.37 & 50.82 \\
\hline CH1015 & SSIM4 & 1074.40 & 26.12 & 1.11 & 51.79 & 164.26 & 100.15 & 42.88 & 14.36 & 121.64 & 31.15 \\
\hline C691 & SSIM4 & 1106.00 & 25.28 & 0.92 & 44.26 & 156.18 & 100.17 & 42.84 & 10.97 & 135.25 & 21.50 \\
\hline C363 & SSIM4 & 1094.50 & 25.17 & 1.02 & 39.61 & 188.74 & 99.79 & 41.26 & 10.89 & 146.49 & 25.08 \\
\hline G656 & SSIM4 & 1027.20 & 26.43 & 2.55 & 217.32 & 25.58 & 102.24 & 41.13 & 14.27 & 149.88 & 50.98 \\
\hline G593 & SSIM4 & 1009.80 & 24.77 & 0.50 & 14.21 & 108.60 & 101.20 & 45.83 & 10.08 & 144.23 & 20.45 \\
\hline
\end{tabular}

5. For each biosynthesis parameter (e.g. $h_{\mathrm{Am}}$,ii) belonging to more than one group, find significant differences 
6. Calculate the group differences between mutant (isoform types) groups by one-way ANOVA

Table 2 The differences between mutant types of starch biosynthesis parameters of SSI isoform.

\begin{tabular}{|c|c|c|c|c|c|c|c|c|c|c|c|c|}
\hline Isoform & $\mathbf{M}$ & No. & $\begin{array}{l}h_{\mathrm{Ap}, \mathrm{i}} \\
\times 10^{3}\end{array}$ & $\begin{array}{r}h_{\mathrm{Ap}, \mathrm{iii}} \\
\times \mathbf{1 0}^{3}\end{array}$ & $\begin{array}{l}h_{\mathrm{Ap}, \mathrm{v}} \\
\times 10^{3}\end{array}$ & $\begin{array}{l}h_{\mathrm{Am}, \mathrm{i}} \\
\times \mathbf{1 0}^{3}\end{array}$ & $\begin{array}{c}\boldsymbol{h}_{\mathrm{Am}, \mathrm{ii}} \times \\
1^{3}\end{array}$ & $\begin{array}{l}\boldsymbol{\beta}_{\text {Ap, }} \mathbf{i} \\
\times 10^{3}\end{array}$ & $\begin{array}{c}\beta_{\text {Ap,iii }} \times \\
10^{3}\end{array}$ & $\begin{array}{l}\beta_{\text {Ap, }, v} \\
\times 10^{3}\end{array}$ & $\boldsymbol{\beta}_{\mathrm{Am}, \mathrm{i}}$ & $\boldsymbol{\beta}_{\mathrm{Am}, \mathrm{ii}}$ \\
\hline \multirow{4}{*}{ SSI } & SSIM1 & 13 & & & & & $109^{\mathrm{a}, \mathrm{b}}$ & & & & $103^{a}$ & \\
\hline & SSIM2 & 43 & & & & & $103^{a}$ & & & & $134^{\mathrm{b}}$ & \\
\hline & SSIM3 & 8 & & & & & $162^{\mathrm{b}}$ & & & & $139^{\mathrm{a}, \mathrm{b}}$ & \\
\hline & SSIM4 & 11 & & & & & $119^{\mathrm{a}, \mathrm{b}}$ & & & & $122^{\mathrm{a}, \mathrm{b}}$ & \\
\hline
\end{tabular}

Note: $\mathrm{M}$ is the mutant group (isoform type group); "No." is the number identification given to that mutant type. The letters $a, b$ represent the statistically significant different between isoform types at $p<0.05$. The statistically valid differences were calculated by one-way ANOVA, to determine whether there are any statistically significant differences between the means of three or more independent (unrelated) groups.

7. Significant differences between mutant groups of any parameters imply that it can be assumed that the isoform could affect those parameters and dominate relative CLDs. 


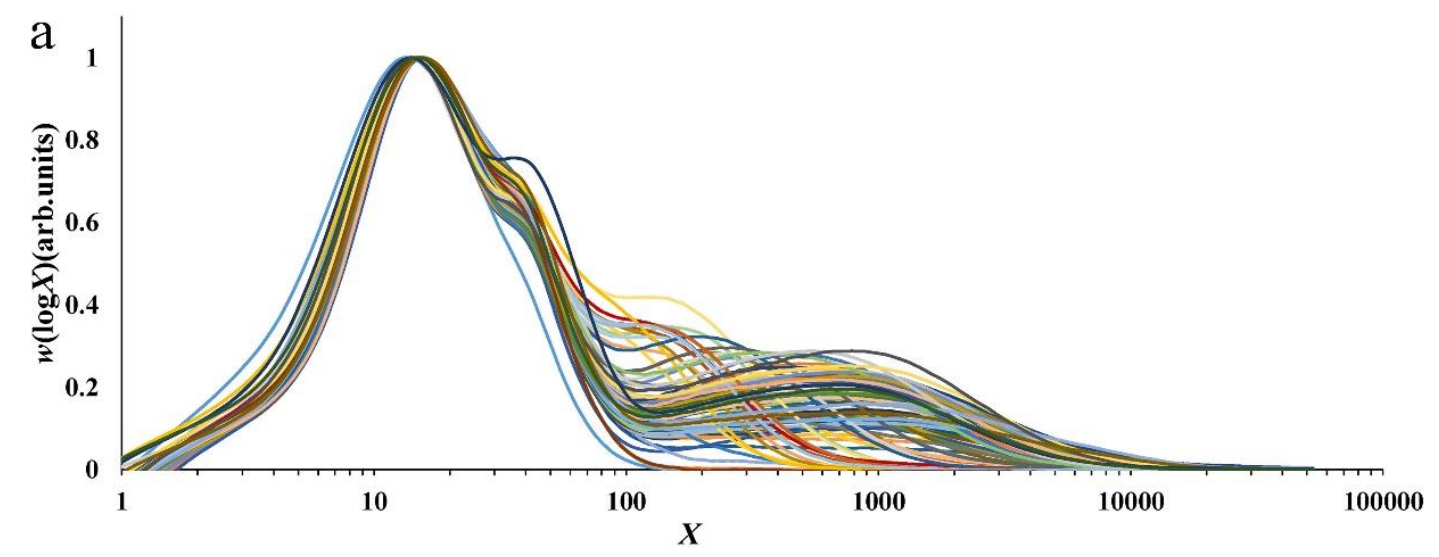

\begin{tabular}{|c|c|c|c|c|c|c|c|c|c|c|}
\hline - C266 & & & & & & & 42 & & & \\
\hline -G239 & - G577 & - $\mathrm{CH} 1269$ & - C1196 & $-\mathrm{G} 194$ & - G562 & - C1038 & $-\mathrm{G} 582$ & 一 SE91 & -SE14 & $-\mathrm{CH} 1114$ \\
\hline - G426 & $-\mathrm{CH} 10$ & $2-\mathrm{G} 224$ & $-\mathrm{C} 1128$ & G528 & $-\mathrm{G} 520$ & $-\mathrm{CH} 121 \mathrm{C}$ & -SE342 & —G593 & $-\mathrm{C} 1031$ & - 9969 \\
\hline -SE223 & -SE108 & - SE344 & $-\mathrm{C} 1170$ & $-\mathrm{CH} 1015$ & SE228 & $-\mathrm{C} 351$ & $-\mathrm{G} 222$ & -SE88 & - G656 & -SE389 \\
\hline - 2287 & - G350 & $-\mathrm{C} 53$ & $-\mathrm{CH} 1117$ & $\mathrm{G} 101$ & $-\mathrm{G} 175$ & G649 & $-\mathrm{C} 1030$ & $-\mathrm{C} 291$ & $-\mathrm{C} 1200$ & $-\mathrm{G} 360$ \\
\hline$-\mathrm{C} 1221$ & $-\mathrm{G} 584$ & $-\mathrm{C} 403$ & $-\mathrm{C} 42$ & $-\mathrm{G} 259$ & $-\mathrm{C} 1061$ & $-\mathrm{C} 263$ & $-\mathrm{CH} 1099$ & -SE217 & $-\mathrm{CH} 126$ & - G561 \\
\hline$-\mathrm{C} 125$ & $-\mathrm{C} 272$ & $-\mathrm{C} 135$ & $-\mathrm{C} 363$ & - SE9 & $-\mathrm{C} 142$ & $-\mathrm{G} 357$ & $-\mathrm{C} 691$ & $-\mathrm{G} 407$ & $-\mathrm{C} 278$ & - SE372 \\
\hline$-\mathrm{C} 1151$ & G182 & $-\mathrm{G} 97$ & CH1148 & $-\mathrm{G} 436$ & $-\mathrm{C} 38$ & $-\mathrm{C} 602$ & $-\mathrm{G} 157$ & $-\mathrm{G} 245$ & $-\mathrm{G} 246$ & \\
\hline
\end{tabular}

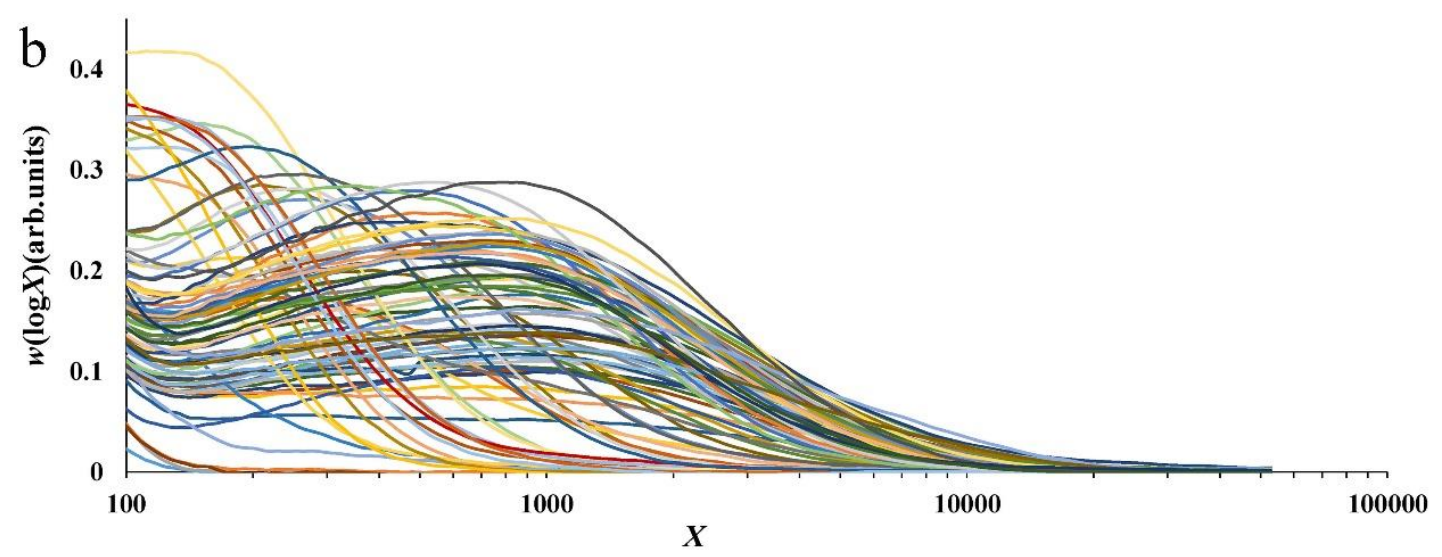

\begin{tabular}{|c|c|c|c|c|c|c|c|c|c|c|}
\hline - C266 & -SE4 & -SE461 & Clo & $-\mathrm{CH}$ & $-\mathrm{CF}$ & - G220 & - 2242 & -G186 & —G546 & -G417 \\
\hline - G239 & $-\mathrm{G} 577$ & $-\mathrm{CH} 1269$ & $-\mathrm{C} 1196$ & G194 & $-\mathrm{G} 562$ & $-\mathrm{C} 1038$ & $-\mathrm{G} 582$ & $\longrightarrow \mathrm{SE} 91$ & $\longrightarrow$ SE14 & $-\mathrm{Cl}$ \\
\hline - G426 & $-\mathrm{CH} 1002$ & $2-\mathrm{G} 224$ & $-\mathrm{C} 1128$ & $\mathrm{G} 528$ & $-\mathrm{G} 520$ & $-\mathrm{CH} 1210$ & $\longrightarrow \mathrm{SE} 342$ & $\longrightarrow \mathrm{G} 593$ & $-\mathrm{C} 1031$ & $-\mathrm{C} 969$ \\
\hline -SE223 & -SE108 & $-\mathrm{SE} 344$ & $-\mathrm{C} 1170$ & $-\mathrm{CH} 1015$ & $-\mathrm{SE} 228$ & $-\mathrm{C} 351$ & $-\mathrm{G} 222$ & $\longrightarrow \mathrm{SE} 88$ & —G656 & -SE389 \\
\hline$-\mathrm{C} 287$ & $-\mathrm{G} 350$ & $-\mathrm{C} 53$ & $-\mathrm{CH}$ & $-\mathrm{G}$ & $-\mathrm{G} 1$ & G649 & $-\mathrm{C} 1$ & $-\mathrm{C} 291$ & $-\mathrm{C} 1200$ & $-\mathrm{G} 3$ \\
\hline$-\mathrm{C} 1221$ & G584 & $-\mathrm{C} 403$ & $-\mathrm{C} 42$ & $-\mathrm{G} 259$ & $-\mathrm{C} 1061$ & $-\mathrm{C} 263$ & $-\mathrm{CH} 1099$ & -SE217 & $-\mathrm{CH} 1263$ & $-\mathrm{G} 561$ \\
\hline$-\mathrm{C} 125$ & $-\mathrm{C} 272$ & $-\mathrm{C} 135$ & $-\mathrm{C} 363$ & $\longrightarrow$ SE9 & $-\mathrm{C} 142$ & $-\mathrm{G} 357$ & $-\mathrm{C} 6$ & $-\mathrm{G} 4$ & $-\mathrm{C} 278$ & -SE372 \\
\hline$-\mathrm{C} 1151$ & - G182 & $-\mathrm{G} 97$ & CH1148 & $-\mathrm{G} 436$ & $-\mathrm{C} 38$ & $-\mathrm{C} 602$ & - G157 & - G245 & - G246 & \\
\hline
\end{tabular}

Supplementary figure 1. SEC weight CLDs, $w(\log X)$, of debranched starches (a) whole DP region, normalized to the maximum of the Ap component and (b) an enlargement of the Am region. 


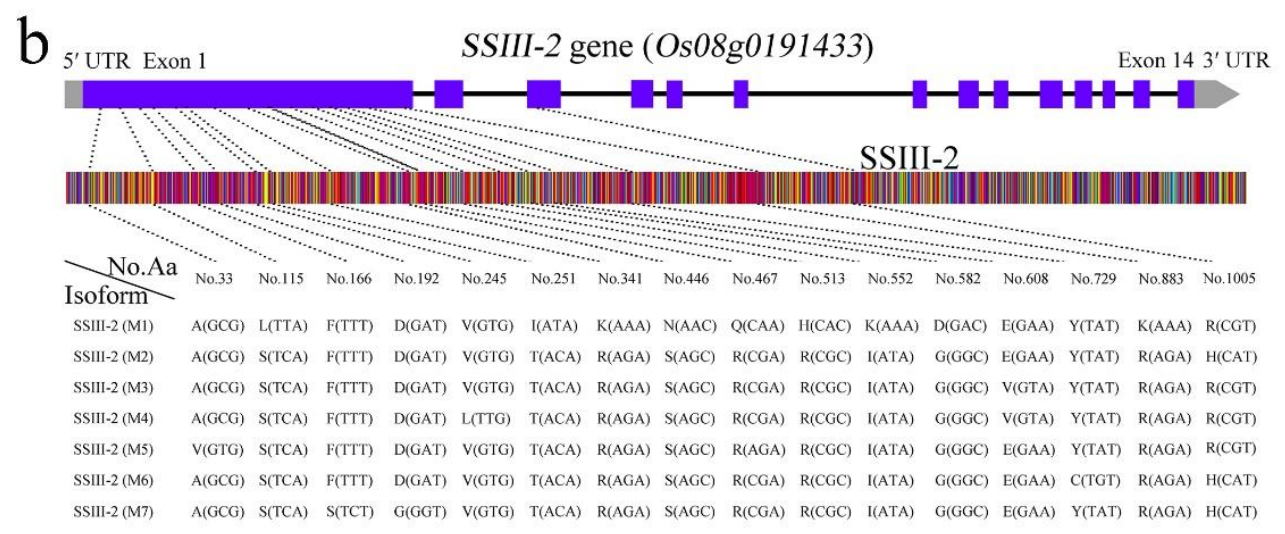

Supplementary figure 2. The gene and enzyme mutant group differences of (a) SSIII-1and (b)

\section{SSIII-2.}


Supplementary Table 187 rice samples used in this study.
Note: M, mutant group; IndI, Indica I; IndII, Indica II; TrJ, Tropical Japonica; TeJ,Temperate Japonica; Aus, early summer rice.

\begin{tabular}{|c|c|c|c|c|c|c|c|c|c|c|c|c|c|c|c|c|}
\hline $\begin{array}{l}\text { Number } \\
01\end{array}$ & Original ID & Name of Species & $\begin{array}{l}\text { Original Country } \\
\end{array}$ & Sub-population & SSIM & $\begin{array}{l}\text { SSII-1 M } \\
\end{array}$ & SSII-2 M & SSII-3 M & $\begin{array}{l}\text { SSIIII-1 M } \\
\end{array}$ & SSIII-2 M & $\begin{array}{l}\text { SSIV-1 M } \\
\end{array}$ & SSIV-2 M & SBEIM & SBEIIa M & SBEIIb M & $\begin{array}{l}\text { GBSSIM } \\
\end{array}$ \\
\hline $\begin{array}{l}\text { S01 } \\
\text { S02 }\end{array}$ & $\begin{array}{l}\text { C38 } \\
\text { C42 }\end{array}$ & $\begin{array}{l}\text { HP 3319-2WX-6-4-1-B } \\
\text { JAERARYUKDO }\end{array}$ & $\begin{array}{l}\text { South Korea } \\
\text { Soutt Korea }\end{array}$ & $\begin{array}{l}\text { IndI } \\
\text { TrJ }\end{array}$ & $\begin{array}{l}1 \\
2\end{array}$ & $\begin{array}{l}4 \\
1\end{array}$ & 2 & 2 & 99 & 5 & 2 & 2 & 1 & 1 & 2 & 3 \\
\hline S03 & $\mathrm{C} 53$ & $\begin{array}{l}\text { JEERAER YUKDO } \\
\text { H 305-84 }\end{array}$ & $\begin{array}{l}\text { South Korea } \\
\text { Hungary }\end{array}$ & TeJ & $\frac{2}{2}$ & $\begin{array}{l}1 \\
3\end{array}$ & $\begin{array}{l}4 \\
4\end{array}$ & $\frac{2}{1}$ & $\begin{array}{l}8 \\
7\end{array}$ & $\frac{2}{3}$ & $\begin{array}{l}4 \\
3\end{array}$ & $\begin{array}{l}2 \\
2\end{array}$ & 1 & 1 & $\begin{array}{l}2 \\
2\end{array}$ & $\begin{array}{l}4 \\
4\end{array}$ \\
\hline S04 & $\mathrm{C} 125$ & ARC 7425 & India & Aus & 3 & 2 & 2 & 3 & 4 & 7 & 2 & 2 & 1 & 1 & 2 & 1 \\
\hline S05 & C135 & AUS 242 & Bangladesh & Aus & & 2 & 3 & 3 & 5 & 6 & 2 & 2 & 2 & 1 & 2 & 1 \\
\hline S06 & $\mathrm{C} 142$ & AUS 329 & Bangladesh & Aus & 3 & 2 & 2 & 3 & 5 & 7 & 2 & 2 & 1 & 1 & 2 & 1 \\
\hline S07 & $\mathrm{C} 263$ & DV 110 & Bangladesh & Aus & & 2 & 2 & 3 & 4 & 7 & 2 & 2 & 2 & 1 & 2 & 1 \\
\hline S08 & C266 & E 2070 & China & IndII & 4 & 2 & 3 & 2 & 8 & 5 & 2 & 2 & 1 & 1 & 1 & 3 \\
\hline S09 & $\mathrm{C} 272$ & EDAKKADAN 0-69-27 & India & IndI & 4 & 4 & 3 & 3 & 8 & 4 & 2 & 2 & 1 & 1 & 2 & 1 \\
\hline$\$ 10$ & C278 & E ZI 100 & China & IndI & 1 & 4 & 3 & 3 & 8 & 5 & 2 & 2 & 1 & 1 & 1 & 2 \\
\hline S11 & C287 & GAO JIAO YING GAN ZHAN & China & IndI & 3 & 4 & 3 & 3 & 3 & 6 & 2 & 2 & 1 & 1 & 2 & 2 \\
\hline$S 12$ & C291 & GENG 77-4 & China & IndII & 1 & 4 & 3 & 2 & - & 1 & 2 & 2 & 1 & 1 & 1 & 2 \\
\hline S13 & C351 & JIANG ER ZAO & China & IndII & 1 & 4 & 3 & 1 & 6 & 7 & 2 & 2 & 1 & 1 & 1 & 2 \\
\hline $\begin{array}{l}\text { S14 } \\
\text { S15 }\end{array}$ & C363 & $\begin{array}{l}\text { KAHATAWEE } \\
\text { KOLAMBA }\end{array}$ & $\begin{array}{l}\text { Sri Lanka } \\
\text { India }\end{array}$ & IndI & 4 & 4 & 3 & 3 & $\begin{array}{l}8 \\
4\end{array}$ & 4 & 2 & 2 & $\frac{1}{2}$ & 1 & $\frac{1}{2}$ & 1 \\
\hline S16 & $\begin{array}{l}\mathrm{C} 403 \\
\mathrm{C} 602\end{array}$ & $\begin{array}{l}\text { KOLAMBA } \\
\text { SALSI }\end{array}$ & $\begin{array}{l}\text { India } \\
\text { India }\end{array}$ & $\begin{array}{l}\text { Aus } \\
\text { Aus }\end{array}$ & $\begin{array}{l}3 \\
3\end{array}$ & 2 & $\begin{array}{l}3 \\
3\end{array}$ & 3 & $\begin{array}{l}4 \\
4\end{array}$ & 7 & 2 & $\frac{2}{2}$ & $\frac{2}{2}$ & 1 & $\frac{2}{2}$ & $\frac{1}{1}$ \\
\hline S17 & C691 & XIANG CHANG ZAO & China & $\begin{array}{l}\text { Inst } \\
\text { IndII }\end{array}$ & 4 & 2 & 3 & 3 & 8 & 5 & 2 & 2 & 1 & 1 & 2 & 2 \\
\hline $\mathrm{S} 18$ & C969 & ZACATEPEC & US & IndI & 1 & 3 & 3 & 2 & 9 & 5 & 2 & 2 & 1 & 1 & 1 & 3 \\
\hline S19 & C1018 & DECHANGBYEO & South Korea & TeJ & 2 & 3 & 4 & 1 & 7 & 1 & 3 & 2 & 1 & 1 & 2 & 3 \\
\hline S20 & C1030 & E ZI 32 & China & IndII & 4 & & 3 & 3 & 9 & 7 & 3 & 2 & 1 & 1 & 1 & 2 \\
\hline S21 & C1031 & FEI ZHAO 12 & China & TeJ & 2 & 3 & 4 & 2 & 7 & 3 & 1 & 2 & 1 & 1 & 2 & 3 \\
\hline S22 & C1038 & GINMASARI & Japan & TeJ & 2 & 3 & 1 & 2 & 7 & 3 & 1 & 2 & 1 & 1 & 2 & 3 \\
\hline S23 & C1061 & KABERI & Bangladesh & IndI & 1 & 4 & 3 & 2 & 1 & 5 & 2 & 2 & 1 & 1 & 2 & 2 \\
\hline S24 & C1128 & OITA MII 120 & Japan & TeJ & 2 & 3 & 4 & 2 & 7 & - & 3 & 2 & 1 & 1 & 2 & 3 \\
\hline S25 & C1151 & 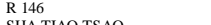 & India & Aus & - & 4 & 2 & 3 & - & - & 2 & 2 & 1 & 1 & 2 & 1 \\
\hline $\begin{array}{l}\text { S26 } \\
\text { S27 }\end{array}$ & C1170 & SHA TIAO TSAO & China & TeJ & 2 & 3 & 4 & 1 & 7 & 3 & 1 & 2 & 1 & 1 & 2 & 4 \\
\hline $\begin{array}{l}\text { S27 } \\
\text { S28 }\end{array}$ & $\begin{array}{l}\text { C1196 } \\
\text { C1200 }\end{array}$ & $\begin{array}{l}\text { YEZO } \\
\text { ZIRA SHAHI }\end{array}$ & $\begin{array}{l}\text { South Korea } \\
\text { Paksista }\end{array}$ & $\begin{array}{l}\text { TeJ } \\
\text { Admix }\end{array}$ & 2 & $\begin{array}{l}3 \\
4 \\
\end{array}$ & 4 & 1 & $\begin{array}{l}7 \\
4\end{array}$ & 3 & 1 & 2 & 1 & 1 & 2 & 3 \\
\hline $\begin{array}{l}\text { S28 } \\
\text { S29 }\end{array}$ & $\begin{array}{l}\mathrm{C} 1200 \\
\mathrm{C} 1221\end{array}$ & $\begin{array}{l}\text { ZIRA SHAHI } \\
\text { IRRI } 146\end{array}$ & $\begin{array}{l}\text { Pakistan } \\
\text { Philippines }\end{array}$ & $\begin{array}{l}\text { Admix } \\
\text { Ind }\end{array}$ & 3 & 4 & 2 & 3 & $\begin{array}{c}4 \\
10\end{array}$ & 4 & 2 & 2 & 1 & 1 & 2 & ${ }_{4}^{1}$ \\
\hline $\begin{array}{l}\mathbf{S} 29 \\
\text { S30 }\end{array}$ & $\begin{array}{l}\text { CH1221 } \\
\text { CH1002 }\end{array}$ & JOGI CHEONGJIN & $\begin{array}{l}\text { Phllpppines } \\
\text { North Korea }\end{array}$ & $\begin{array}{l}\text { Indi } \\
\text { TeJ }\end{array}$ & $\begin{array}{l}2 \\
2\end{array}$ & 3 & 4 & $\begin{array}{l}3 \\
2\end{array}$ & ${ }_{7}^{10}$ & $\begin{array}{l}4 \\
3\end{array}$ & $\frac{2}{3}$ & $\begin{array}{l}2 \\
2\end{array}$ & $\begin{array}{l}1 \\
1\end{array}$ & 1 & $\begin{array}{l}1 \\
2\end{array}$ & $\begin{array}{l}4 \\
3\end{array}$ \\
\hline S31 & CH1015 & LUA SOM & Vietnam & IndII & 4 & 4 & 3 & & 9 & 7 & 2 & 2 & 1 & 1 & 1 & 2 \\
\hline S32 & CH1063 & K 24 & India & IndII & 1 & 4 & 3 & 1 & 9 & 7 & 2 & 2 & 1 & 1 & 1 & 2 \\
\hline S33 & CH1091 & 80050 YR 72136-43 & Australia & TrJ & 2 & 1 & 4 & 1 & 7 & 3 & 3 & 2 & 1 & 1 & 1 & 3 \\
\hline S34 & CH1099 & ERJIU NAN 1 & China & IndI & 4 & 4 & 3 & 2 & 3 & 5 & 2 & 2 & 1 & 1 & 2 & 2 \\
\hline S35 & CH1114 & BAO XIE 123B & China & IndI & & 4 & 3 & 3 & & & 2 & 2 & 1 & 1 & 1 & 3 \\
\hline S36 & CH1117 & LONG HUA MAO HU & China & TeJ & 2 & 3 & 4 & 1 & 7 & 1 & 3 & 2 & 1 & 1 & 2 & 1 \\
\hline S37 & CH1148 & BA WANG BIAN 1 & China & Admix & 2 & 3 & 4 & 2 & 6 & 3 & 3 & 2 & 1 & 1 & 2 & 3 \\
\hline $\mathbf{S} 38$ & CH1210 & SHU FENG 101 & China & IndII & 4 & 2 & 3 & 3 & 10 & 7 & 2 & 2 & 1 & 1 & 1 & 2 \\
\hline$S 39$ & CH1263 & ZHU ZHEN B & China & IndII & - & 4 & 3 & 1 & 10 & 7 & 2 & 2 & 1 & 1 & 1 & 2 \\
\hline$S 40$ & CH1269 & QING SI AI 16B & China & IndI & - & 3 & 3 & 3 & 1 & 5 & 2 & 2 & & 1 & 1 & 3 \\
\hline $\begin{array}{l}\mathrm{S} 41 \\
\mathrm{~S} 42\end{array}$ & $\begin{array}{l}\text { G97 } \\
\text { G101 }\end{array}$ & $\begin{array}{l}\text { A 201 } \\
\text { ARIANA }\end{array}$ & $\begin{array}{l}\text { US } \\
\text { Romania }\end{array}$ & $\begin{array}{l}\text { TrJ } \\
\text { IndI }\end{array}$ & $\frac{2}{2}$ & $\begin{array}{l}3 \\
4\end{array}$ & 4 & 1 & ${ }_{10}^{1}$ & $\begin{array}{l}3 \\
4\end{array}$ & 3 & 2 & $\begin{array}{l}3 \\
1\end{array}$ & 1 & 1 & $\begin{array}{l}1 \\
4\end{array}$ \\
\hline $\begin{array}{l}\mathbf{S} 42 \\
\mathbf{S} 43\end{array}$ & $\begin{array}{l}\text { Gio1 } \\
\text { G157 }\end{array}$ & $\begin{array}{l}\text { AROANA } \\
\text { IBO } 400\end{array}$ & $\begin{array}{l}\text { Romanaa } \\
\text { Portugal }\end{array}$ & $\begin{array}{l}\text { Indal } \\
\text { TeJ }\end{array}$ & $\begin{array}{l}2 \\
2\end{array}$ & $\begin{array}{l}4 \\
4\end{array}$ & $\begin{array}{l}3 \\
4\end{array}$ & $\begin{array}{l}3 \\
2\end{array}$ & ${ }_{7}^{10}$ & $\begin{array}{l}4 \\
2\end{array}$ & $\begin{array}{l}2 \\
1\end{array}$ & $\begin{array}{l}2 \\
2\end{array}$ & 1 & 1 & $\begin{array}{l}1 \\
2\end{array}$ & $\begin{array}{l}4 \\
3\end{array}$ \\
\hline S44 & G175 & IRAT 335 & Bolivia & TrJ & 2 & 4 & 4 & 1 & 10 & 7 & 4 & 2 & 1 & 1 & 2 & 4 \\
\hline S45 & G182 & L 205 & US & TrJ & 3 & 1 & 4 & 1 & 1 & 2 & 3 & 2 & 1 & 1 & 1 & 2 \\
\hline S46 & G186 & LOTO & Italy & TeJ & 2 & 3 & 1 & 2 & 7 & 3 & 1 & 2 & 1 & 1 & 2 & 3 \\
\hline S47 & G194 & MELAS & Greece & TeJ & 2 & 1 & 1 & 2 & 1 & 3 & 3 & 2 & 1 & 1 & 2 & 3 \\
\hline S48 & G220 & ROXANI & Greece & TeJ & 2 & 3 & 4 & 1 & 7 & 1 & 1 & 1 & 1 & 1 & 2 & 3 \\
\hline S49 & G222 & RUBIDIO & Italy & TrJ & 2 & 1 & 4 & 2 & 7 & 3 & 1 & 2 & 1 & 1 & 1 & 4 \\
\hline S50 & G224 & RUBINO & Italy & TeJ & 2 & 1 & 4 & 2 & 7 & 3 & 1 & 2 & 1 & 1 & 2 & 3 \\
\hline S51 & G239 & SMERALDO & Italy & TeJ & 2 & 1 & 1 & 2 & 7 & 3 & 1 & 2 & 1 & 1 & 2 & 3 \\
\hline$S 52$ & G242 & SR 113 & Spain & TeJ & 2 & 1 & 1 & 2 & 7 & 3 & 1 & 1 & 1 & 1 & 2 & 3 \\
\hline $\begin{array}{l}\text { S53 } \\
\text { S54 }\end{array}$ & G245 & $\begin{array}{l}\text { THAIPERLA } \\
\text { TIMICH }\end{array}$ & US & TeJ & 2 & 3 & 4 & 2 & 7 & 3 & 1 & 2 & 1 & 1 & 1 & 3 \\
\hline $\begin{array}{l}\text { S54 } \\
\text { S55 }\end{array}$ & $\begin{array}{l}\text { G246 } \\
G 259\end{array}$ & $\begin{array}{l}\text { TIMICH } 108 \\
\end{array}$ & Romania & TeJ & 2 & 3 & 4 & 2 & 7 & 3 & 3 & 2 & 1 & 1 & 2 & 3 \\
\hline $\begin{array}{l}\text { S55 } \\
\text { S56 }\end{array}$ & $\begin{array}{l}\text { G259 } \\
\text { G350 }\end{array}$ & $\begin{array}{l}\text { WAS 199-B-1-2-1 } \\
\text { BLUE BELE }\end{array}$ & $\begin{array}{l}\text { Senegal } \\
\text { US }\end{array}$ & $\begin{array}{l}\text { IndI } \\
\text { TrJ }\end{array}$ & 2 & $\begin{array}{l}4 \\
1\end{array}$ & 3 & 3 & 10 & 5 & ${ }_{4}^{2}$ & 2 & 1 & 1 & 2 & $\begin{array}{l}1 \\
4\end{array}$ \\
\hline $\begin{array}{l}\mathbf{S} 56 \\
\text { S57 }\end{array}$ & $\begin{array}{l}\text { Gi350 } \\
\text { G357 }\end{array}$ & $\begin{array}{l}\text { BLUE BELLE } \\
\text { CAUVERY }\end{array}$ & $\begin{array}{l}\text { India } \\
\text { Ind }\end{array}$ & $\begin{array}{l}\text { Ind } \\
\text { IndI }\end{array}$ & $\begin{array}{l}2 \\
1\end{array}$ & $\begin{array}{l}1 \\
4\end{array}$ & 3 & $\begin{array}{l}1 \\
3\end{array}$ & 1 & $\begin{array}{l}3 \\
4\end{array}$ & $\begin{array}{l}4 \\
2\end{array}$ & $\begin{array}{l}2 \\
2\end{array}$ & 1 & $\begin{array}{l}1 \\
1 \\
\end{array}$ & $\begin{array}{l}1 \\
2\end{array}$ & $\begin{array}{l}4 \\
1\end{array}$ \\
\hline S58 & G360 & CHANDINA & Sri Lanka & IndI & 4 & 4 & 3 & 3 & 1 & 5 & 2 & 2 & 1 & 1 & 1 & 1 \\
\hline S59 & G407 & GENIT & Argentina & Admix & - & 2 & 2 & 3 & 8 & 4 & 2 & 2 & 1 & 1 & 2 & 1 \\
\hline S60 & G417 & HOKURIKU 52 & Japan & TrJ & - & 1 & 4 & 1 & 1 & 2 & 3 & 2 & 3 & 1 & 2 & 4 \\
\hline S61 & G426 & IRI 339 & South Korea & IndI & 1 & 4 & 3 & 2 & & 5 & 2 & 2 & 1 & 1 & 1 & 3 \\
\hline S62 & G436 & KALIN & Bulgaria & TeJ & 2 & 3 & 4 & 1 & 7 & 3 & 1 & 2 & 1 & 1 & 2 & 3 \\
\hline S63 & G520 & S 201 & US & TeJ & 2 & 3 & 4 & 2 & 7 & - & 1 & 2 & 1 & 1 & 1 & 3 \\
\hline S64 & G528 & SKY BONNET & US & TrJ & 2 & 1 & 4 & 1 & 1 & 2 & 4 & 2 & 1 & 1 & 2 & 4 \\
\hline S65 & G546 & TONG GU HONG & China & IndI & 3 & 4 & 3 & 3 & 2 & 5 & 2 & 2 & 1 & 1 & 1 & 2 \\
\hline S66 & G561 & YONG AN HUK & South Korea & TeJ & 2 & 3 & 4 & 2 & 7 & 3 & 3 & 2 & 1 & 1 & 2 & 3 \\
\hline S67 & G562 & YONG IINZAO 3 & China & IndII & 1 & 2 & 3 & 1 & 9 & 7 & 2 & 2 & 1 & 1 & 1 & 2 \\
\hline $\begin{array}{l}\mathbf{S} 68 \\
\text { S69 }\end{array}$ & $\begin{array}{l}\text { G577 } \\
\text { G582 }\end{array}$ & $\begin{array}{l}\text { ECIA 24-107-1 } \\
\end{array}$ & $\begin{array}{l}\text { Cuba } \\
\text { Guatemal }\end{array}$ & $\begin{array}{l}\text { IndI } \\
\text { Ind }\end{array}$ & 2 & 4 & 3 & 2 & 8 & 7 & 2 & 2 & 1 & 1 & 1 & 3 \\
\hline $\begin{array}{l}S 69 \\
\$ 70\end{array}$ & $\begin{array}{l}\text { G582 } \\
\text { G584 }\end{array}$ & $\begin{array}{l}\text { ICTA CRISPO } 38 \\
\text { INAP } 10\end{array}$ & $\begin{array}{l}\text { Guatemala } \\
\text { Fcundor }\end{array}$ & $\begin{array}{l}\text { IndI } \\
\text { IndI }\end{array}$ & 2 & 4 & 3 & 2 & $\begin{array}{l}6 \\
8 \\
8\end{array}$ & $\frac{3}{5}$ & 2 & $\begin{array}{l}2 \\
2\end{array}$ & 1 & 1 & 1 & 3 \\
\hline S71 & G593 & IR $73690-7-2-1-1-1-3-2-2-1$ & $\begin{array}{l}\text { Ecuador } \\
\text { Philippines }\end{array}$ & $\begin{array}{l}\text { IndI } \\
\text { Aus }\end{array}$ & 4 & $\begin{array}{l}4 \\
4\end{array}$ & $\begin{array}{l}3 \\
2\end{array}$ & $\frac{2}{3}$ & $\begin{array}{l}8 \\
5\end{array}$ & $\begin{array}{l}3 \\
6\end{array}$ & $\begin{array}{l}3 \\
2\end{array}$ & $\frac{2}{2}$ & $\begin{array}{l}1 \\
1\end{array}$ & $\begin{array}{l}1 \\
1\end{array}$ & $\begin{array}{l}1 \\
2\end{array}$ & $\frac{2}{1}$ \\
\hline S72 & G649 & RUSTIC & Guyana & IndI & 2 & & 3 & 2 & 10 & 5 & 4 & 2 & 1 & 1 & 1 & 1 \\
\hline S73 & G656 & CHANG LE SAN SHU ZAO & China & IndII & 4 & 4 & 3 & 3 & 9 & 7 & 2 & 2 & 1 & 1 & 1 & 2 \\
\hline S74 & SE4 & 93072 & China & Admix & & & & 2 & & & & 2 & & 1 & 1 & 3 \\
\hline S75 & SE9 & FENG AI ZHAN & China & IndI & - & 4 & 3 & 2 & 6 & 7 & 2 & 2 & 1 & 1 & - & \\
\hline S76 & SE14 & CHEN NONG 265 & China & TeJ & 2 & 3 & 4 & 2 & 7 & 3 & 1 & 2 & 1 & 1 & 2 & 3 \\
\hline$\$ 77$ & SE88 & M 202 & US & IndI & & 4 & 3 & & & $=$ & 2 & 2 & 1 & 1 & - & - \\
\hline S78 & SE91 & UPR 191-66 & India & IndI & 2 & 4 & 3 & 2 & 8 & 5 & 2 & 2 & 1 & 1 & 1 & 3 \\
\hline 579 & SE108 & DACCA 6 & India & TeJ & 2 & 1 & 1 & 1 & 8 & 3 & 1 & 1 & 1 & 1 & 1 & 4 \\
\hline $\begin{array}{l}S 80 \\
S 81\end{array}$ & SE217 & FL 478 & Philippines & IndI & 1 & 4 & 3 & 3 & 3 & 5 & 2 & 2 & 1 & 1 & 1 & 2 \\
\hline S81 & $\begin{array}{l}\text { SE223 } \\
\text { SEF? }\end{array}$ & IR 77298-14-1-2 & Philippines & IndI & 2 & & 3 & 3 & & 7 & 2 & 2 & 1 & 1 & 1 & 4 \\
\hline $\begin{array}{l}\text { S82 } \\
S 83\end{array}$ & $\begin{array}{l}\text { SE228 } \\
\text { SE342 }\end{array}$ & $\begin{array}{l}\text { IR 64-IL } \\
\text { YUNGENG } 23\end{array}$ & Philippines & IndI & 2 & 4 & 3 & 3 & 2 & 3 & 2 & 2 & 1 & 1 & 1 & 4 \\
\hline $\begin{array}{l}\begin{array}{l}S 83 \\
\text { S84 }\end{array}\end{array}$ & $\begin{array}{l}\text { SE342 } \\
\text { SE344 }\end{array}$ & $\begin{array}{l}\text { YUN GENG } 23 \\
\text { SAGC-7 }\end{array}$ & $\begin{array}{l}\text { China } \\
\text { China }\end{array}$ & $\begin{array}{l}\text { TeJ } \\
\text { IndI }\end{array}$ & $\frac{2}{2}$ & $\begin{array}{l}3 \\
4\end{array}$ & ${ }_{3}^{4}$ & 2 & $\begin{array}{l}7 \\
7\end{array}$ & 2 & $\begin{array}{l}3 \\
2\end{array}$ & $\frac{2}{2}$ & $\begin{array}{l}1 \\
1\end{array}$ & $\begin{array}{l}1 \\
1\end{array}$ & $\begin{array}{l}2 \\
1\end{array}$ & $\frac{3}{3}$ \\
\hline $\begin{array}{l}584 \\
\text { S85 }\end{array}$ & SE372 & NERICA-L-1 & Cote d'IVoire & IndI & 2 & 4 & 3 & 3 & 2 & - & 2 & 2 & 1 & 1 & 1 & \\
\hline S86 & SE389 & SARD & NONE & IndI & 1 & 4 & 3 & 2 & 10 & 7 & 2 & 2 & 1 & 1 & 1 & 3 \\
\hline$\$ 87$ & SE461 & TONGNIAN 1 & China & TeJ & 2 & 3 & 4 & 2 & 7 & 3 & 3 & 2 & 1 & 1 & 2 & 3 \\
\hline
\end{tabular}


Supplentary Table 2: Single base mutation and amino acid mutation of isoforms of starch biosynthesis enzymes Note: IRGSP, International Rice Genome Sequencing Project; Aa, amini acid.

\begin{tabular}{|c|c|c|c|c|c|c|c|c|c|}
\hline Isoform & Gene & strand & Chr. & IPGSP-1.0 position & Exon & Nip base & Mutation base & Nip Aa & Mutation Aa \\
\hline \multirow{7}{*}{ SSI } & \multirow{7}{*}{ Os06g0160700 } & \multirow{7}{*}{-} & \multirow{7}{*}{6} & 3082326 & 9 & $\mathrm{~A}$ & $\mathrm{G}$ & $\mathrm{K}$ & $\mathrm{E}$ \\
\hline & & & & 3082490 & 8 & $\mathrm{C}$ & $\mathrm{T}$ & $\mathrm{H}$ & $\mathrm{Y}$ \\
\hline & & & & 3083079 & 6 & A & $\mathrm{G}$ & $\mathrm{S}$ & $\mathrm{G}$ \\
\hline & & & & 3086385 & 1 & G & $\mathrm{T}$ & A & $\mathrm{S}$ \\
\hline & & & & 3086397 & 1 & A & $\mathrm{G}$ & $\mathrm{T}$ & $\mathrm{A}$ \\
\hline & & & & 3086439 & 1 & $\mathrm{C}$ & A & $\mathrm{L}$ & M \\
\hline & & & & 3086531 & 1 & $\mathrm{G}$ & $\mathrm{T}$ & $\mathrm{R}$ & $\mathrm{L}$ \\
\hline \multirow{3}{*}{ SSII-1 } & \multirow{3}{*}{ Os $10 \mathrm{~g} 0437600$} & \multirow{3}{*}{+} & \multirow{3}{*}{10} & 15673341 & 1 & $\mathrm{C}$ & $\mathrm{T}$ & $\mathrm{A}$ & $\mathrm{V}$ \\
\hline & & & & 15675357 & 4 & $\mathrm{G}$ & A & $\mathrm{R}$ & $\mathrm{H}$ \\
\hline & & & & 15675473 & 4 & $\mathrm{G}$ & $\mathrm{T}$ & A & $\mathrm{S}$ \\
\hline \multirow{5}{*}{ SSII-2 } & \multirow{5}{*}{ Os02g0744700 } & \multirow{5}{*}{-} & \multirow{5}{*}{2} & 31234046 & 9 & $\mathrm{C}$ & $\mathrm{T}$ & $\mathrm{L}$ & $\mathrm{F}$ \\
\hline & & & & 31237132 & 3 & $\mathrm{~T}$ & $\mathrm{C}$ & $\mathrm{S}$ & $\mathrm{P}$ \\
\hline & & & & 31237203 & 3 & A & $\mathrm{C}$ & $\mathrm{Y}$ & $\mathrm{S}$ \\
\hline & & & & 31237662 & 2 & G & A & V & M \\
\hline & & & & 31237695 & 2 & A & $\mathrm{G}$ & $\mathrm{T}$ & $\mathrm{A}$ \\
\hline \multirow{2}{*}{ SSII-3 } & \multirow{2}{*}{ Os06g0229800 } & + & 6 & 6752357 & 8 & $\mathrm{~A}$ & $\mathrm{G}$ & $\mathrm{S}$ & $\mathrm{G}$ \\
\hline & & + & 0 & 6752888 & 8 & $\mathrm{C}$ & $\mathrm{T}$ & $\mathrm{L}$ & $\mathrm{F}$ \\
\hline & & & & 31752269 & 16 & $\mathrm{~A}$ & $\bar{G}$ & $\mathrm{~T}$ & $\mathrm{~A}$ \\
\hline & & & & 31752654 & 14 & $\mathrm{C}$ & $\mathrm{G}$ & $\mathrm{L}$ & V \\
\hline & & & & 31753447 & 11 & $\mathrm{C}$ & $\mathrm{T}(\mathrm{C})$ & $\mathrm{S}$ & $\mathrm{S}(\mathrm{N})$ \\
\hline & & & & 31753448 & 11 & G & $\mathrm{G}(\mathrm{A})$ & $\mathrm{S}$ & $S(I N)$ \\
\hline & & & & 31755480 & 6 & $\mathrm{G}$ & A & V & I \\
\hline & & & & 31755497 & 6 & $\mathrm{G}$ & $\mathrm{T}$ & $\mathrm{S}$ & I \\
\hline SSIII-1 & Os04g0624600 & - & 4 & 31756405 & 5 & A & G & $\mathrm{E}$ & $\mathrm{G}$ \\
\hline & & & & 31757436 & 3 & G & A & $\mathrm{R}$ & $\mathrm{H}$ \\
\hline & & & & 31757746 & 3 & $\mathrm{C}$ & $\mathrm{T}$ & $\mathrm{L}$ & $\mathrm{F}$ \\
\hline & & & & 31758136 & 3 & $\mathrm{G}$ & A & $\mathrm{E}$ & $\mathrm{K}$ \\
\hline & & & & 31758185 & 3 & $\mathrm{~A}$ & $\mathrm{C}$ & $\mathrm{K}$ & $\mathrm{N}$ \\
\hline & & & & 31758208 & 3 & G & $\mathrm{T}$ & V & $\mathrm{F}$ \\
\hline & & & & 31758390 & 3 & $\mathrm{~T}$ & $\mathrm{G}$ & $\mathrm{F}$ & $\mathrm{C}$ \\
\hline & & & & 5353938 & 1 & $\mathrm{C}$ & $\mathrm{T}$ & $\mathrm{A}$ & $\mathrm{V}$ \\
\hline & & & & 5354184 & 1 & $\mathrm{C}$ & $\mathrm{T}$ & $\mathrm{S}$ & $\mathrm{L}$ \\
\hline & & & & 5354337 & 1 & $\mathrm{~T}$ & $\mathrm{C}$ & $\mathrm{F}$ & $\mathrm{S}$ \\
\hline & & & & 5354415 & 1 & A & $\mathrm{G}$ & $\mathrm{D}$ & $\mathrm{G}$ \\
\hline & & & & 5354573 & 1 & $\mathrm{G}$ & $\mathrm{T}$ & $\mathrm{V}$ & $\mathrm{L}$ \\
\hline & & & & 5354592 & 1 & $\mathrm{C}$ & $\mathrm{T}$ & $\mathrm{T}$ & I \\
\hline & & & & 5354862 & 1 & $\mathrm{G}$ & A & $\mathrm{R}$ & $\mathrm{K}$ \\
\hline & & & & 5355177 & 1 & $\mathrm{G}$ & A & $\mathrm{S}$ & $\mathrm{N}$ \\
\hline SSIII-2 & Os08g0191433 & + & 8 & 5355239 & 1 & $\mathrm{C}$ & $\mathrm{A}(\mathrm{C})$ & & \\
\hline & & & & 5355240 & 1 & G & $\mathrm{G}(\mathrm{A})$ & $\mathrm{R}$ & $\mathrm{R}(\mathrm{Q})$ \\
\hline & & & & 5355378 & 1 & $\mathrm{G}$ & A & $\mathrm{R}$ & $\mathrm{H}$ \\
\hline & & & & 5355495 & 1 & $\mathrm{~T}$ & A & I & $\mathrm{K}$ \\
\hline & & & & 5355585 & 1 & G & A & $\mathrm{G}$ & $\mathrm{D}$ \\
\hline & & & & 5355663 & 1 & $\mathrm{~T}$ & A & $\mathrm{V}$ & $\mathrm{E}$ \\
\hline & & & & 5356026 & 1 & A & $\mathrm{G}$ & $\mathrm{Y}$ & $\mathrm{C}$ \\
\hline & & & & 5356488 & 1 & $\mathrm{G}$ & $\mathrm{A}$ & $\mathrm{R}$ & $\mathrm{K}$ \\
\hline & & & & 5357570 & 5 & G & A & $\mathrm{R}$ & $\mathrm{H}$ \\
\hline & & & & 30035044 & 11 & $\bar{G}$ & $\mathrm{~A}$ & $\bar{G}$ & $\mathrm{D}$ \\
\hline & & & & 30038156 & 5 & $\mathrm{~T}$ & $\mathrm{C}$ & V & A \\
\hline SSIV-1 & Os01g0720600 & - & 1 & 30038502 & 4 & $\mathrm{~T}$ & A & $\mathrm{S}$ & $\mathrm{T}$ \\
\hline & & & & 30038819 & 4 & $\mathrm{~A}$ & $\mathrm{G}$ & $\mathrm{H}$ & $\mathrm{R}$ \\
\hline & & & & 30039379 & 4 & $\mathrm{G}$ & $\mathrm{T}$ & $\mathrm{L}$ & $\mathrm{F}$ \\
\hline SSIV-2 & Os05g0533600 & + & 5 & 26492492 & 13 & $\mathrm{G}$ & $\mathrm{A}$ & $\mathrm{D}$ & $\mathrm{N}$ \\
\hline SBEI & $0 \mathrm{~s} 06 \mathrm{o} 0726400$ & - & 6 & 30898818 & 13 & $\mathrm{C}$ & $\mathrm{T}$ & $\mathrm{A}$ & $\mathrm{V}$ \\
\hline SDEI & Usuoguizo400 & - & 0 & 30900768 & 7 & $\mathrm{~A}$ & $\mathrm{C}$ & $\mathrm{I}$ & $\mathrm{L}$ \\
\hline SBEIIb & Os02g0528200 & - & 2 & 19365137 & 4 & $\mathrm{~A}$ & $\mathrm{G}$ & $\mathrm{H}$ & $\mathrm{R}$ \\
\hline & & & & 1765761 & 5 ' intron 1 & $\mathrm{G}$ & $\mathrm{T}$ & - & - \\
\hline GBSSI & Os06g0133000 & + & 6 & 1768006 & 6 & A & $\mathrm{C}$ & $\mathrm{Y}$ & $\mathrm{S}$ \\
\hline & & & & 1768998 & 10 & $\mathrm{C}$ & $\mathrm{T}$ & $\mathrm{P}$ & $\mathrm{S}$ \\
\hline
\end{tabular}

